\title{
Supporting offline activities on interactive surfaces
}

\author{
Augusto Esteves ${ }^{1}$, Michelle Scott ${ }^{1,2}$, Ian Oakley ${ }^{1,2}$ \\ ${ }^{1}$ Exact Sciences and Engineering Center, University of Madeira, Funchal, Portugal \\ ${ }^{2}$ Madeira Interactive Technologies Institute, Funchal, Portugal \\ augustoeae@gmail.com; mscott@m-iti.org; ian.r.oakley@gmail.com
}

\begin{abstract}
This paper argues that inherent support for offline activities - activities that are not sensed by the system - is one of strongest benefits of tangible interaction over more traditional interface paradigms. By conducting two studies with single and paired users on a simple tangible tabletop scheduling application, this paper explores how tabletop interfaces could be designed to better support such offline activities. To focus its exploration, it looks at offline activities in terms of how they support cognitive work, such as aiding exploration of problem spaces or lowering task complexity. This paper concludes with insights relating to the form, size, and location for spaces that afford offline actions, and also the design of tangible tokens themselves.
\end{abstract}

\section{Author Keywords}

Tangible interaction, offline activities, embodied cognition.

\section{ACM Classification Keywords}

H5.m. Information interfaces and presentation (e.g., HCI): Miscellaneous.

\section{General Terms}

Design, Human factors.

\section{INTRODUCTION}

Tangible interaction connects multiple physical forms to digital representations, allowing users to interact with data through their hands and bodies using physical objects (or tokens) as "interface, interaction object and interaction device" [18]. Proponents of the tangible paradigm have ascribed it various benefits, including improved usability; increased levels of engagement and enjoyment; effectiveness is learning scenarios; and seamless support for collaboration [30]. A key factor underlying these prospective benefits is the integration of tangible interaction with the physical world, such that users are able to apply their naïve, but highly refined real world understandings of physical systems to interaction in the digital domain [19].

This idea is compelling. However, embeddedness in the world has additional implications. For example, recent

Permission to make digital or hard copies of all or part of this work for personal or classroom use is granted without fee provided that copies are not made or distributed for profit or commercial advantage and that copies bear this notice and the full citation on the first page. To copy otherwise, or republish, to post on servers or to redistribute to lists, requires prior specific permission and/or a fee.

TEI 2013, Feb 10-13, 2013, Barcelona, Spain.

Copyright 2013 ACM 978-1-4503-1898-3/13/02...\$15.00. work has highlighted the inevitability (and associated potential for confusion and discord) of manipulations of tangible objects that take place offline [e.g. 14, 17] beyond the scope a system's ability to sense them. In tabletop systems, for instance, how users organize and manage objects beyond the surface's plane is reported to substantially impact user behavior. Examples include helping to seamlessly mediate behaviors such as token passing in collaborative play [31], or creating confusion and frustration when metaphors between object manipulation in the real and digital worlds breaks down [17].

This paper argues that offline activities represent a practically important and potentially rich design space capable of supporting a range of high-level tasks. Compelling examples illustrating this point exist. For instance, in Durrell-Bishop's seminal Marble Answering Machine [1] glass beads linked to voicemail messages could be placed up in physically customizable passive containers associated with specific family members or activities (see Figure 1). Similarly, in Mementos [13], simply selecting a set of tokens to use with the system was characterized as a collaborative decision making process, while the large number of tokens in the LogJam Video Logging system [9] encouraged users to systematically structure and organize tokens away from the sensing surface. Taken together these examples highlight the potential value and diversity of designing for offline manipulations of tangible elements, but also the piecemeal nature of research on this topic.

Inspired by this work, this paper suggests that offline activities represent an important, unique and unexplored aspect of tangible systems. It seeks to provide an early description of the impact of such activities through the development and evaluation of Eco Planner [12], a simple tabletop application that requires the use and management of a large token set. Two studies of the system were conducted covering individual and paired system use. The qualitative and quantitative results from these experiments were synthesized into a set of design insights that focuses on form, size, and location of the repositories for offline tokens. These insights are intended to motivate and frame future research efforts to create a more comprehensive body of knowledge regarding how to design tangible interaction to support what this paper argues is one of its inevitable and fundamental characteristics: offline activity, or the use of tokens as the natural physical objects they actually are. 

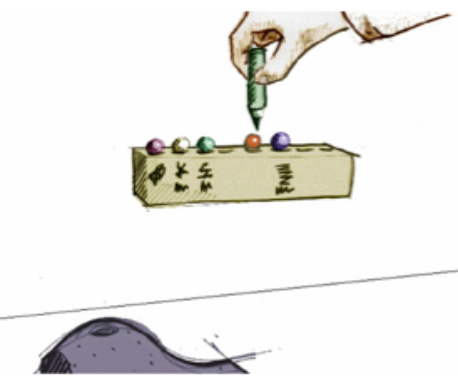

Figure 1. One of the possible offline activities in the Marble Answering Machine [1]: using tokens outside of the system to tag them according to caller.

\section{RELATED WORK}

Tangible systems provide "tangible representations to digital information and controls, allowing users to quite literally grasp data with their hand and effect functionality by physical manipulations of these representations" [30]. The undeniable appeal and promise of such fluid, naturalistic properties has led to the development of tangible research prototypes in domains as diverse as education, entertainment, and data management [30]. Although quantifying the benefits of the tangible paradigm has proven challenging [35], typical goals are to promote learning through physical engagement [7], to leverage real physical metaphors for control [4], or to support collaboration by lowering thresholds for participation [16].

However, as Dourish points out, one unique and powerful property of embodied, physical objects is that they naturally encourage ad-hoc use, customization and personalization [11]. These activities typically take place offline, beyond the sensing scope of a tangible system. Dourish argues that supporting such manipulations is a key design goal for the creation of truly embodied digital artifacts capable of participating in the activities they support. But determining how this participation can be practically achieved in the design of a tangible system remains a challenging task.

Embodied cognition [34] is an emerging perspective in cognitive science that emphasizes the role of body and environment in everyday tasks of perception, comprehension, and problem solving. For instance, Clark [8] discusses external scaffolding, or the tendency for people to offload cognitive work from their minds to the environment and the mechanisms used to achieve this. Everyday examples include leveraging the persistent and consistent nature of the physical world as a surrogate for memory (as in Chipchase's center of gravity, a zone in the home in which objects to take to work are piled up each evening), and in the distinction between epistemic and pragmatic actions [22]. The former refers to altering the world to aid, augment or replace cognitive work (e.g. rotating a Tetris piece to better see where it fits) while the latter denotes activity to actually complete a task (e.g. maneuvering a Tetris piece into place). Literature suggests that epistemic actions support both immediate task completion and skill acquisition [3].

Such theoretical understandings have great practical value and have recently been used to inform the design of a number of tangible systems. For instance, MoSo Tangibles [6], a set of interactive tokens to teach sonic concepts such as pitch and tempo explores interaction via direct manipulation of embodied concepts such as speed, size and height. Similarly, NOOT [10], a tangible application to support group brain-storming activities characterizes the external output (post-its, diagrams, or sketches) as external scaffolding and seeks to augment this material digitally by providing physical markers and handles for audio clips.

Little work, however, has systematically studied or described the offline uses of tangible tokens and how understandings of embodied activity can inform their design. Systems such as the Marble Answering Machine [1], Mementos [13] and LogJam [9] argue that passive tokens are appropriate targets for annotations, can aid collaborative decision making and also be profitably structured and organized by users. However, such efforts are ad-hoc and do not seek to systematically explore this design space. The goal of this paper is to start to address this lack by seeking to better characterize and understand how users work with tokens as passive objects. It focuses on the offline management of large token sets and how this can be effectively supported in the design of tangible systems. In particular, it is concerned with the design of token repositories, or zones in which tokens can be cached when out of use, and identifies three dimensions on which these can meaningfully vary: form, size and location.

\section{ECO PLANNER}

Eco Planner was developed to explore the design of repositories for offline tokens. Four requirements guided the development: the need to deal with a broadly applicable and understandable problem domain - representing a set of activities on a grid or calendar-like schedule (as in SenseBoard [20]); the need to support a large token set, ensuring that users would have to manage both online and offline tokens; the need to provide users with different forms of repository for offline tokens; and the need to support collaboration by allowing for multiple tokens to be used simultaneously and for the overall task to be meaningful as a group activity.

In Eco Planner users position iconic tokens on a grid to create routines representing their activities in the home [12]. Its focus is on creating and visualizing activities that involve energy consumption in order to calculate and understand the environmental impact of particular behavior patterns and consumption choices. It is composed of a set of physical tokens that are set up on an interactive tabletop surface. Via iconic labels, each token represents a specific activity (e.g., watching TV, doing the laundry), and users create their household routines by configuring these objects 
on the tabletop's surface. The application provides real time feedback and recommendations relating to the objects arranged on its surface, including an overall score representing resources consuming by the entire schedule. Users can to set the system to display these cues as either ecological or financial messages (e.g. by displaying the $\mathrm{CO}^{2}$ footprint or expenses accumulated).

The Eco Planner interface is shown in Figure 2. The interface is divided in three major areas. The routine area takes the form of a large rectangular zone that represents a single day (from $7 \mathrm{am}$ to $11 \mathrm{pm}$ ). Time is along the horizontal (or long) axis - tokens placed on the left of the routine area represent activities conducted in the morning, while tokens on the right represent activities in the evening. Tokens aligned vertically (or along the short axis) represent concurrent activities. It also features an options area, a small central zone where users can position tokens, and interact with a simple interface that lets them commit to different behaviors relating to that activity. For instance, with the laundry object, users can choose to commit to always doing the laundry with a full load of clothes.

To explore how spatial consistency in the repositories for offline tokens may influence how users interact and manage them while not altering the system's state, two versions of Eco Planner were developed: labeled and blank. In the labeled version (Figure 2), Eco Planner has four colorcoded repositories, each marked as a particular area of the home (e.g. kitchen, bedroom). These labels provide users with a coherent and consistent place to store offline tokens, e.g. they are able to stash tokens representing kitchen activities in the kitchen zone, thus supporting subsequent easy access for themselves or other users. In the blank version, the four repositories have a single color (grey), providing users with no clues in how they should organize their offline tokens. In both cases, the repository zones were situated at the corners of the tabletop, sites where users have previously been observed storing unused tokens [29].

Eco Planner was implemented using the Processing programming language, and runs on a tabletop system constructed using the Diffused Surface Illumination (DSI) method, the ReacTIVision tracking software, and the TUIO messaging protocol. The interactive surface was 78 by 57 centimeters in size and composed of a diffusing layer $(5 \mathrm{~mm}$ thick Evonik ACRYLITE 7D006) placed on top of a sensing layer (10mm thick Evonik Endlighten). The sensing layer was wrapped with a string of $850 \mathrm{~nm}$ IR diodes, with a near throw projector and IR camera positioned underneath the plastics such that both could address the full area of the interactive surface. A DSI setup is a simple and reliable way to track both fiducial markers and fingertips on an interactive tabletop. The tokens used in the system were 7 by 7 by 2.5 centimeters wooden cuboids with iconic labels affixed to their uppermost surface and ReacTIVision markers on their base. 27 tokens were deployed in the Eco Planner system, representing 13 unique activities.

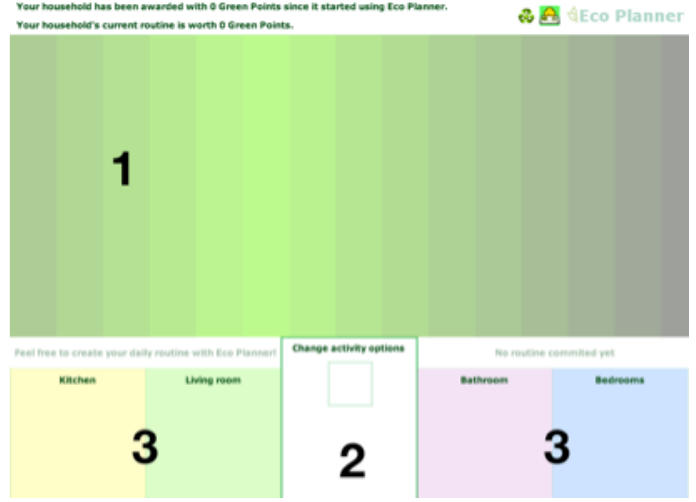

Figure 2. The Eco Planner interface: (1) the routine area, where users can spatially lay tokens to create their daily routines; (2) the options area, where users can commit to a series of changes to a particular activity; and (3) the repository area, where offline tokens can be rested.

\section{METHOD}

This section describes a study of participants interacting with the Eco Planner tabletop system. It followed a between groups design with three independent variables: collaboration (single/paired), to explore how offline tokens might be used to support group activities; initial token configuration (local/distant), to explore how the initial configuration of offline tokens cues and influences users; and repository zone format (labeled/blank), to explore to how much users rely on such cues for spatial consistency when engaging in offline activities.

The collaboration and token configuration variables were tied together such that single participants used distant tokens and paired participants used local tokens. Repository zone format was distributed equally across these two groups: half of the single/distant group and half of the paired/local group used the labeled repositories and half used the blank repositories. The collaboration variable was adjusted by recruiting two participant pools, individual and pairs of users, as described in the participants' section of this paper. Paired users were required to complete the experimental tasks collaboratively. The repository zone variable was modulated via enabling the two zone types in the Eco Planner interface as required. Finally, token configuration was varied via changes to the experimental procedure, as noted in the procedure section.

\section{Participants}

Single users: A total of 12 single participants were recruited, eight males and four females. There were five Portuguese, three Indians, two Venezuelans, an American, and a Pakistani participant. Their ages ranged from 23 to 35 $(\mathrm{M}=26.5, \mathrm{SD}=4.2)$ and all were students or full time research staff at our research institute.

Paired users: Six pairs of participants were recruited (four males, eight females) and each pair of participants had lived 
together (for a minimum six months) at the time of the study. There were four participants from the USA, two Taiwanese, two Portuguese, a Canadian, a Swede, a Kenyan, and an Indian participant. Their ages ranged from 23 to $33(\mathrm{M}=26.2, \mathrm{SD}=2.9)$, and all of them were either undergraduate or postgraduate students at our university. One pair of participants was a couple, whereas the other five pairs were cohabitants (flat/room mates).

\section{Procedure}

The experiment started with participants being shown the tabletop display and receiving instructions on how to operate Eco Planner. All participants were then asked to model three different routines. The first two were derived from short written scenarios depicting two fictional households, while the final routine was based on participants' own activities at home and aimed at exploring differences in behavior for more experienced users. Participants had total freedom over defining the routines. There was no time limit for the tasks and at the end of the experiment participants filled in a paper questionnaire.

The experimental procedures varied according to two types of initial token configuration. In the distant condition, an experimenter set up the tokens on a desk a few meters from the Eco Planner tabletop. Participants were required to choose a set of tokens from the desk and bring them to the tabletop prior to interaction. After each routine was completed the tokens were returned to the distant desk. The goal of this set up was to explore how users would configure the offline tokens in the available repositories on the tabletop, without any influences from predefined token positions. On the other hand, in the local condition, the tokens were initially set up in the repository areas of the Eco Planner system and tokens were not returned to preset positions in-between the experimental tasks. Participants were required to manage the tokens themselves as they created the three different routines. The goal of this setup was to explore how much users rely or are constrained by the initial spatial configuration of offline tokens, with special attention to this process in a collaborative setting.

\section{Measures}

Measures were obtained from video recordings of participants interacting with the tabletop. The analysis was formalized and objective data coded and extracted by a single researcher. To gain insight into how effective the offline tokens and their repositories were in supporting embodied thought, two times were extracted:

- Hold time. The mean duration a token is in the hand of a user, measured from pick up until put down. This included time spent moving the token.

- Search time: The mean duration between holding tokens. This is the time from when a participant places a token down until they pick one up again.
These measures were used as proxies for the amount of time it took to decide on an appropriate course of action after picking up a token and for the amount of time it took to locate the appropriate tokens from repository areas. These measures will help determine if the strategies that users employ users to resolve the Eco Planner tasks are actually the strategies that allow them to perform more rapidly and efficiently. To reduce variance in this data, it was filtered using a selection criterion: if users were judged to be distracted (e.g. starting a discussion with another user), or commenced another action while holding or searching for a token, the measurement was discarded. After the completion of each of the three tasks, the total number of tokens used in the solution was recorded.

Qualitative data was also inferred from the videos, in order to explore the strategies developed by each participant (or pair of participants) to organize and move the offline tokens from the repositories to the sensable interface areas of the application (see Figure 2). In the collaborative condition, verbal and physical communications (e.g. pointing, touching, guiding or token passing) between participants were also noted, in order to establish whether participants were adopting distinctive roles as discussed by Shaer et al. [31]. Finally, subjective data was also captured. A brief semi-structured interview followed the study and asked participants to expand on their strategies for managing the offline tokens during the experiment.

\section{RESULTS}

In this section general data taken from both quantitative and qualitative measurements is presented. This is done to better understand what strategies users adopted when interacting with the system in terms of: actions undertaken; number of tokens used (both online and offline); and chosen repositories for offline activities. Experimental results are in Table 1.

The first finding is that, for single users, the duration of actions stays relatively steady or decreases as they become more familiar with the system (search time: $F(2)=3.71, p$ $<0.05$; hold time: $F(2)=2.98, p=0.07)$, whereas the number of tokens used remained fairly constant throughout (Task 1: 7.83/2.73; Task 2: 8.08/3.68; Task 3: 7.50/1.88). Despite previous work showing that experienced users are more likely to engage in offline activities (e.g. epistemic actions) [22], these results point out that it is not always the case, with factors such as task complexity or time pressure influencing in how much cognitive support will users seek from offline tokens.

While there was no significant differences between the duration of actions performed by single and paired users, the latter group used more tokens than the former (Task 1: $t(22)=-5.83, p<0.001$; Task 3: $t(22)=-9.05, p<0.001)$. Lastly, single users were also faster than paired users in searching for tokens $(t(21)=-3.56, p<0.01)$, but also took longer to decide what to do with them (hold time). 


\begin{tabular}{|c|c|c|c|}
\hline & Task 1 & Task 2 & Task 3 \\
\hline \multirow{2}{*}{$\begin{array}{c}\text { Search } \\
\text { time }\end{array}$} & Single: $2.87(1.40)$ & Single: 2.37 (1.06) & Single: 3.06 (1.90) \\
\hline & Pair: 4.49 (1.73) & Pair: 4.57 (2.55) & Pair: 5.25 (2.48) \\
\hline \multirow{2}{*}{$\begin{array}{l}\text { Hold } \\
\text { time }\end{array}$} & Single: $2.03(0.73)$ & Single: 2.47 (1.15) & Single: $1.54(0.44)$ \\
\hline & Pair: 2.37 (1.65) & Pair: 2.19 (1.26) & Pair: $1.66(0.47)$ \\
\hline \multirow{2}{*}{$\begin{array}{c}\text { Tokens } \\
\text { used }\end{array}$} & Single: 7.83 (2.74) & Single: 8.08 (3.68) & Single: 7.50 (1.88) \\
\hline & Pair: 13.50 (1.98) & Pair: 8.80 (1.55) & Pair: 13.50 (1.31) \\
\hline
\end{tabular}

Table 1. Mean times for search, hold, and number of tokens used in the routine created. Standard deviation in brackets (single and paired users).

\section{Strategies}

The strategies employed in problem solving tasks provide insights into participants' cognitive activities. By using offline tokens to lower task complexity, users create a spatial structure where they know what to expect, where to look, and what to look for [27]. Three distinct strategies of interaction emerged from the observation of the participants tackling each of the different tasks. The first strategy, repository-focus (R), was characterized by users who relied heavily on the repositories for offline tokens throughout the task. Such users would pick a token from the repositories, explore the available options (via the options zone of the interface, see Figure 2), and, if satisfied, lay the token in the appropriate location in the routine area of the interface. Alternatively, they would either reject the token (or cue it for later exploration) by returning it to a repository area. In the second strategy, action-focus (A), participants would move tokens directly from the repositories to the routine area, laying out routines from ideas established when the tokens were in the repositories. In the end, they would pick each token from the routine area and adjust the options in turn. The last strategy, mixed-focus (M), merged both R and A strategies.

Additionally, participants in the distant condition adopted three repositories to deal with tokens (see Figure 3):

- Interaction space (IS): participants would rest the tokens directly in the tabletop's routine area (online);

- Hand (H): participants would hold offline tokens in their hands and arms, sometimes creating a queue system to simplify token access and choice;
- Periphery (P): participants would use either the edges of the tabletop or repository area built into the interface to hold offline tokens. In one particular case, a participant brought a nearby office chair close to the system so that he could better organize the tokens there.

As participants in the distant condition become more proficient with the system they converged on strategy A and repository $\mathrm{H}$ (7 out of 12). The same was observed for participants in the local condition (who were primed by the setup to use the repository areas to hold offline tokens), where 8 out 12 pairs used strategy A in Task 3. For these last participants, strategy $M$ was the most popular for the first two tasks, with strategy R becoming less popular as users become more experienced with the system (it was not used in Task 3 at all). Additionally, participants in the distant condition opted for the hand repository in all of the tasks (Task 1: used by 6 out of 12 participants; Task 2: used by 9 out of 12; and, Task 3: used by 7 out of 12).

\section{DISCUSSION}

As Klemmer states: "We possess nearly unlimited modes of interaction with the physical world" [23]. Through tangible interaction, users are now able to interact with digital resources through physical tools that are available to them in the real world. But human behavior is complex and subject to a wide range of influencing factors [28]. As such, and as a result of a continuous interaction with the surrounding environment, users develop their own strategies to deal with the problems at hand. But due to the array of available options at any point during interaction, such strategies might not even be the most 'efficient' in objective terms [8]. It is clear that providing users with such openness in terms of how they interpret their interaction with technology creates a new challenge for developers [15]. This paper argues that this is one of the greatest challenges when designing tangible applications - how to accommodate not only task oriented actions, but also offline activities that complement task completion. This challenge extends beyond design to include evaluation of such systems, as offline activities are both poorly defined and hard to predict - there is little literature suggesting what objective offline activities to look for [15]. This ultimately makes these activities hard to formally capture and represent using quantitative methods from HCI [28].
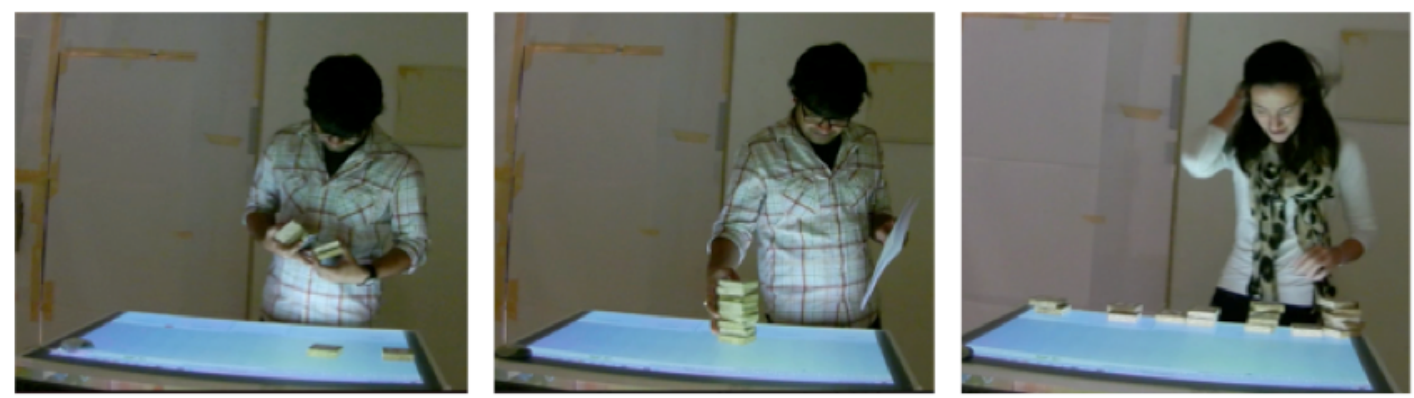

Figure 3. Three repositories that naturally emerged from participants in the distant condition (from left to right): hand $(\mathrm{H})$, interactive space (IS), and periphery $(\mathrm{P})$. 
Addressing this lack, this paper presents initial work exploring the design of interactive tabletop systems to support offline activities, especially those that aimed at offloading cognition into the environment to decrease task complexity. Although the study presented focuses on a particular task, the insights gathered on offline activities are intended to be broad enough to be useful and applicable to a wide range of future systems. As such, unlike traditional guidelines in $\mathrm{HCI}$ that focus on specific interaction mechanisms [e.g. 5, 26], what is proposed are insights into how to support a broader set of activities that are relevant to the rapidly growing paradigm of tangible interaction. These are presented next, and cover three different areas of interaction design: the form of repositories for offline tokens; their size; and their location in a tabletop system. These areas are intended to serve as directions for future research that aims to study or support offline activity.

Repository form: The study manipulated the form of the available token repositories: a single, large, differentiated area versus four smaller, meaningfully categorized zones. Such distinctions appear in the literature (for example in the Marble Answering Machine's [1] use of a single large cache for message-beads integrated into the machine itself in conjunction with individually labeled dishes on which to stash and aggregate specific groups of beads) and a key goal of the current study was to explore how users take advantage of such spaces. The results indicate the zones were largely ignored. In $61 \%$ of the tasks in the condition in which participants were required to bring relevant objects to the table, the arms and hands were used as the primary storage space for the tokens. Similarly, in the condition when the tokens were appropriately set up in the repositories prior to the study, participants rarely used the labeling on the zones to support their tasks. In contrast, they grouped or stacked the tokens according to their own schemes or mental structures [3] (typically based on token similarity) during breaks between tasks.

There are a number of possible accounts for this behavior. Explaining the tendency to hold tokens in their hands, several participants reported uncertainty about the function of the repositories - they formed a part of the tabletop (e.g. in that the graphical contents were digitally projected) and the behavior of the system to tokens in the repository was perceived to be ambiguous. A clear recommendation is therefore that repositories need be clearly marked and possibly even visibly beyond the active sensing and display areas of a tangible system. When tokens were already present in the repositories users were much more likely to take advantage of these zones suggesting that such clear examples are very useful in communicating appropriate behaviors to novice users of tangible systems. The use of physical affordances (e.g. fixtures such as racks that are clearly designed to contain and store tokens [e.g. 1]) may be a useful mechanism by which to achieve this. However, despite using the zones, participants typically ignored the categorical structures in place in the four-zone condition in favor of their own schemes. This observation supports Dourish's notions that customization and appropriation are key to offline activity [11] and suggests that the design of repositories might be best directed towards supporting these forms of personalization as opposed to establishing predetermined structures or categories.

Repository size: Users relying on the repository areas of the interface were observed de-stacking tokens whenever space was available, attempting to ensure that the greatest number of tokens was always visible simultaneously. Furthermore, although participants in the local condition (in which most used the in-hand repository) were faster than participants in the distant condition (where all used the repository areas) at locating desired tokens, the latter created more elaborate and complex solutions. This comes as no surprise, as people naturally seek to augment their cognitive abilities by, for example, reorganizing their environment [33]. Spatial rearrangement aids problem solving by changing how users look at a problem, making objects within the task more perceptible [18]. This can be further explained through a particular kind of external "scaffolding" [8] introduced by Kirsh as projection [21]. Kirsh suggests that projection is cognitive work that mixes perception (which relies on sensed information) with imagination (which relies on cognitive information) to yield mental augmentations that are anchored to physical artifacts. As such, knowing how much space should be reserved for offline activities is important, as it influences how many tokens are perceivable by the users which in turn impacts on how they think and plan for the task at hand.

However, a substantial drawback of tangible interaction is that physical tokens inevitably create clutter [30], so repository size affects how flexibly this can be contained. A consideration of the activities that repositories need support can therefore provide insights into the size they should adopt. The results for this study indicate that the space for offline activities needs be as large as possible - ideally sufficient to clearly layout the full token set in the system. However, designers need consider not only the number of tokens in their system but also the nature of the tasks users will perform. For example, many board games (e.g. chess) mandate the use of only one offline token at a time. The current study also suggests that users rely on repositories in the initial stages of learning a task. This can be observed with both experienced single and paired users, as they heavily relied on strategy A - quickly creating a routine that would give them a basic context to refine and develop. This suggests that the areas of a system reserved for offline activities might not be as useful for experienced users.

However, in collaborative situations, this effect lessened. Indeed, paired users were slower to decide which token to use, but also held tokens for shorter periods of time. This seems to suggest that the decision making process takes place when the tokens are offline, in the repository areas. This conclusion makes sense if we consider that the offline 
areas of the interface are ideal sites for multiple users to debate and discuss how to tackle the task at hand. The fact that paired users used more tokens than single users further supports this idea. As such, it seems that tangible systems supporting multiple users should offer more extensive repository areas than systems for single users, even if employing the same number of tokens.

Repository location: Another important issue to consider in the design of repositories for offline tokens is where they should be placed. One of the hallmarks of tangible interaction is its capacity to support collaboration by, e.g., providing users with multiple access points to tokens, lowering the threshold for participation [16] and enabling such objects to act as resources for shared activity [14]. Repositories need be selected to reflect such behaviors.

In terms of collaboration in the current study, paired users were observed taking two distinct but interchangeable roles. One would act on the tabletop, laying tokens in their final positions and deciding on which options to set-up. The other would take a supporting role, by choosing the next token to be used, providing verbal or visual tips (e.g. pointing), or discussing possibilities and scenarios. This an interaction profile previously identified by Shaer et al. [31] as the driver-navigator (see Figure 4). This suggests that while it is the physicality of tangible interaction that enables a clear understanding between users [23], the idea that multiple individuals will interact similarly, simultaneously and synergistically on a tabletop does not actually apply to real tasks. As such, the ideal areas to support offline activities do not necessarily need to be perfectly accessible to every user in the system: in many cases, users will naturally adopt asymmetric partnerships and repositories should support these roles. Moreover, as it was shown that both single and paired experienced users tended to converge to a common strategy, conducting preliminary studies over prototype systems seems like an ideal way to reveal candidate locations for repository areas.

\section{CONCLUSIONS AND FUTURE WORK}

The current study has a number of limitations in its ability to highlight repository use. This is to be expected, as designing for and evaluating offline use is a challenging endeavor. For instance, the task and experimental setting may be too short or simple to elicit a full range of behaviors. As such, future work in the tangible interaction community is needed to more rigorously explore the preliminary findings presented. Initial efforts should seek to develop an improved understanding of the impact of spaces for offline activities on users' performance, and how this varies with the number of tokens in a system, the nature of the task (e.g. problem solving, spatial manipulation) and what kind of epistemic actions are afforded (e.g. stacking). This last point includes investigations of repositories designed with physical constraints to support specialized actions such as piling or stacking. Additionally, an important focus for future research would be in supporting collaborative users adopting a range of roles. If these roles can be identified via technologies that track users [e.g. 2], then customized dynamic feedback supporting and aiding offline use could be provided. For instance, a "driver" might receive information regarding key areas of the interface to work on, while a "navigator" might get feedback on the location of tokens spread around the table surface. An additional limitation of the current work was that both the interaction style and repository areas of the system constrained users to operate from one side of the tabletop. This was not a major issue in this study, as two participants could both stand side-by-side comfortably and also easily reach any area on the surface. However, it would be interesting to observe how users rely on their orientation towards the tabletop in their organization of offline tokens.

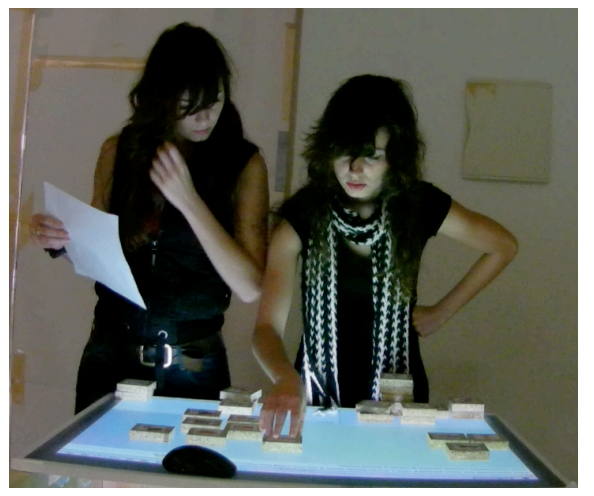

Figure 4. Two participants engaging with the system in what Shaer et al. [31] driver-navigator collaboration profile.

Another important area for future work is on the design of tangible tokens themselves. They are at the core of the tangible interaction paradigm and it is their physical nature that allows for the persistent, non-digital associations that enable their use outside a system's sensing scope. This paper argues that if tokens are to be used in offline activities, they should be designed in order to support a set of epistemic actions (e.g. grouping, queuing, stacking). However, future work is required to flesh out this recommendation. This paper speculatively suggests the following qualities should be explored: to be stackable or attachable to one another; to be adherent to the system's periphery and sides (e.g. through magnets); and to be easy to hold, queue and sort on the users' bodies (e.g. in hands or balanced on an arm). Additionally, tokens need maintain visibility, so that they can meaningfully signify data even when grouped. Such design ideas will help support small, flexible and practically useful repository areas.

In conclusion, it has been shown that a small change in an interactive tabletop's size or shape can have a dramatic impact on its use [32]. While not comprehensively answering why this is the case, this paper contributes to the field of tangible interaction by suggesting that changes in support for offline activities may be partly responsible for this. Indeed, such activities are a unique aspect of tangible 
systems and only by examining such distinctive properties will we be able to elucidate the real benefits and value of the tangible paradigm. This paper takes small steps in that direction; its discussion points the way for future work in the field to illuminate this path further.

\section{REFERENCES}

1. Abrams, R. 1999. Adventures in tangible computing: The work of interaction designer 'Durrell Bishop' in context. Master's thesis, RCA, London.

2. Annett, M., Grossman, T., Wigdor, D., Fitzmaurice, G. 2011. Medusa: a proximity-aware multi-touch tabletop. In Proc. of UIST '11.

3. Antle, A.N. 2012. Exploring how children use their hands to think: An embodied interactional analysis. Behaviour and Information Technology, in press.

4. Antle, A.N. 2007. The CTI framework: informing the design of tangible systems for children. Proc. of TEI'07.

5. Apple Computer, Inc. 1992. Macintosh Human Interface Guidelines. Reading, MA: Addison-Wesley Publishing Company. ISBN 0-201-62216-5.

6. Bakker, S., Hoven, E., van den, Antle, A.N. 2010. MoSo tangibles: evaluating embodied learning. In Proc. of TEI'11.

7. Bruner, J.S. 1966. Toward a theory of instruction. Harvard University Press, Cambridge, MA, USA.

8. Clark, A. 2001. Reasons, Robots and the Extended Mind. Mind \& Language 16(2):121-145.

9. Cohen, J., Withgott, M., Piernot, P. 1999. Logjam: a tangible multi-person interface for video logging. In Proc. of CHI' 99.

10. Dijk, J., van, Roest, J., van der, Lugt, R., van der, Overbeeke, K. 2011. NOOT: a tool for sharing moments of reflection during creative meetings. In Proc. of $C \& C^{\prime} 11$.

11. Dourish, P. 2001. Where the Action Is: the foundations of Embodied Interaction. Cambridge: MIT.

12. Esteves, A. and Oakley I. 2011. Eco Planner: A Tabletop System for Scheduling Sustainable Routines. ACM TEI, Funchal, Portugal, January 23 - 26, 139-144.

13. Esteves, A. and Oakley, I. 2010. Mementos: a tangible interface supporting travel. In Proc. of NordiCHI'10.

14. Fernaeus, Y., Tholander, J., Jonsson, M. 2008. Beyond representations: Towards an action-centric perspective on tangible interaction. I. J. Arts and Tech.

15. Fernaeus, Y., Tholander, J., Jonsson, M. 2008. Towards a new set of ideals: consequences of the practice turn in tangible interaction. In Proc. of TEI '08.

16. Horn, M.S., Solovey, E. T., Crouser, R. J., Jacob, R. J. K. 2009. Comparing the use of tangible and graphical programming interfaces for informal science education. In Proc. of CHI '09.
17. Hornecker, E. 2012. Beyond affordance: tangibles' hybrid nature. In Proc. of TEI'12.

18. Hornecker, E. and Buur, J. 2006. Getting a grip on tangible interaction: a framework on physical space and social interaction. In Proc. of CHI '06.

19. Jacob, R.J., Girouard, A., Hirshfield, L.M., Horn, M.S., Shaer, O., Solovey, E.T., Zigelbaum, J. 2008. Realitybased interaction: a framework for post-WIMP interfaces. In Proc. of SIGCHI' 08.

20. Jacob, R.J., Ishii, H., Pangaro, G., Patten, J. 2002. A tangible interface for organizing information using a grid. In Proc. of CHI '02.

21. Kirsh, D. 2009. Projection, Problem Space and Anchoring. In Proc. of Cognitive Science Society.

22. Kirsh, D. and Maglio, P. 1994. On distinguishing epistemic from pragmatic actions. Cognitive Science.

23. Klemmer, S.R., Hartmann, B., Takayama, L. 2006. How bodies matter: five themes for interaction design. In Proc. of DIS '06.

24.Lin, A., Gregor, S., Ewing, M. 2008. Developing a scale to measure the enjoyment of Web experiences. Journal of Direct Marketing 22 (4), 40-57.

25. Matthews, B. 2006. Grammar, meaning and movementbased interaction. In Proc. of $\mathrm{OzCHI}$ '06.

26. Newman, W. and Lamming, M. 1995. Interactive System Design. Addison Wesley.

27. Petre, M. 1995. Why looking isn't always seeing: readership skills and graphical programming. Commun. ACM 38, 6 (June), 33-44.

28. Procter, R. and Williams, R. 1992. HCI: Whose problem is it anyway? In Proc. of EHCI '92.

29. Scott, S.D., Sheelagh, M., Carpendale, T., Inkpen, K.M. 2003. Territoriality in Collaborative Tabletop Workspaces. In Proc. of CSCW'03.

30. Shaer, O. and Hornecker, E. 2010. Tangible User Interfaces: Past, Present, and Future Directions. Found. Trends Hum.-Comput. Interact. 3, 1- 2 (January).

31. Shaer, O., Strait, M., Valdes, C., Feng, T., Lintz, M., Wang, H. 2011. Enhancing genomic learning through tabletop interaction. In Proc. of CHI'11.

32. Toney, A. and Thomas, B.H. 2006. Applying reach in direct manipulation user interfaces. Proc. of $\mathrm{OzCHI}$ '06.

33. Weller, A., Villejoubert, G., Vallée-Tourangeau, F. 2011. Interactive insight problem solving. Thinking \& Reasoning, 17(4), 424-439.

34. Wilson, M. 2002. Six views of embodied cognition. Psychonomic Bulletin \& Review, 9.

35.Zaman, B., Abeele, V., Markopoulos, P., Marshall, P. 2011. Editorial: the evolving field of tangible interaction for children: the challenge of empirical validation. Personal Ubiquitous Comput. 16, 4 (April), 367-378. 